\title{
Glottic Malignant Fibrous Histiocytoma: A Case Report and Literature Review
}

\author{
Adham A. Aljariria Abdulrahman R. Alsaleh ${ }^{a}$ Hussain A. Al-Enazi ${ }^{a}$ \\ Hasan A. Haider ${ }^{a}$ Mahir Petkar $^{b}$ Waheed Rahman $^{a}$ \\ Abdulqadir Jeprel Nashwanc, d \\ atolaryngology Department, Ambulatory Care Center (ACC), Hamad Medical Corporation \\ (HMC), Doha, Qatar; ${ }^{b}$ Pathology Department, Hamad General Hospital (HGH), Hamad \\ Medical Corporation (HMC), Doha, Qatar; ' $H a z m$ Mebaireek General Hospital (HMGH), \\ Hamad Medical Corporation (HMC), Doha, Qatar; dUniversity of Calgary in Qatar (UCQ), \\ Doha, Qatar
}

\section{Keywords}

Laryngeal cancer - Sarcoma - Laryngectomy - Undifferentiated pleomorphic sarcoma ·

Malignant fibrous histiocytoma

\section{Abstract}

Pleomorphic sarcoma of the larynx is a rare variant of laryngeal cancer. We present the case of a 59-year-old male patient who has been smoking for 40 years. He presented with signs and symptoms of an obstructive glottic mass. The diagnostic workup pointed to a malignant pathology; the histopathology report confirmed the diagnosis of glottic undifferentiated pleomorphic sarcoma (malignant fibrous histiocytoma).

\section{Introduction}

More than 170,000 patients are diagnosed with laryngeal cancer and up to 90,000 deaths occur each year; this accounts for $1-5 \%$ of all cancers and deaths annually [1, 2]. Squamous cell carcinoma accounts for $85-95 \%$ of all laryngeal cancers. It is more common in males, and multiple risk factors have been identified, tobacco and alcohol being mostly related. Most laryngeal cancers arise from the squamous epithelium, while a small percentage develops from other laryngeal tissues $[1,3,4]$. 


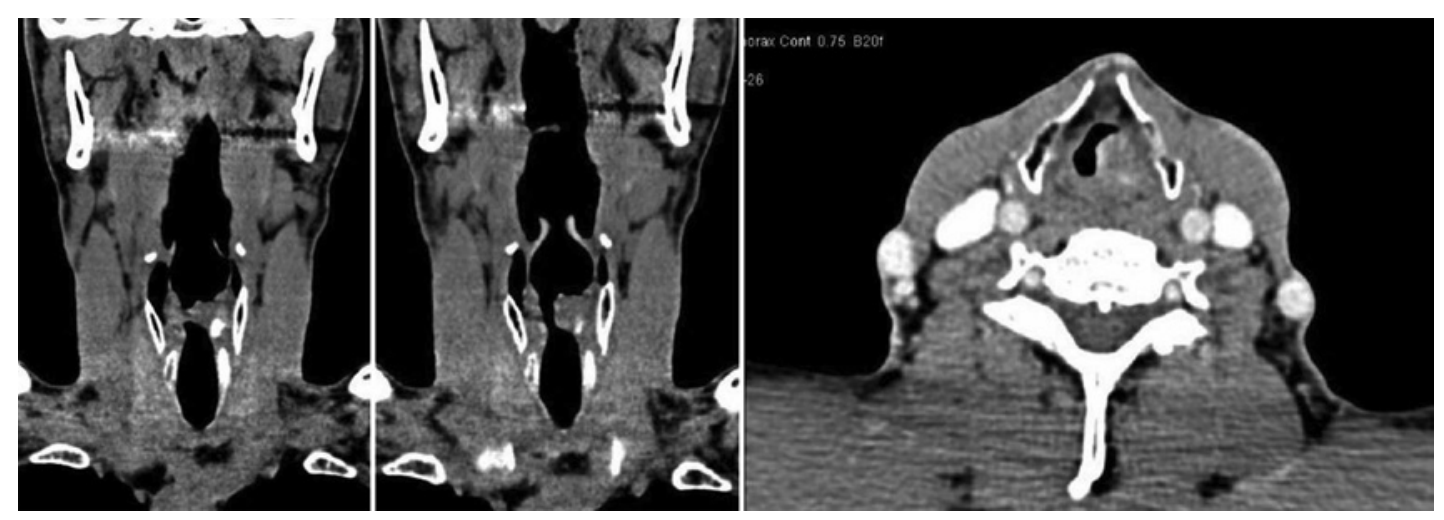

Fig. 1. Coronal and axial neck CT scan with contrast.

Radiotherapy, endolaryngeal excision, and open surgery are all accepted modalities in the treatment of early-stage glottic cancers, with endolaryngeal excision and radiotherapy having the advantage of voice function preservation. Recently, trends are shifting from open surgery toward organ preservation intervention, as there is no significant difference in survival between radiotherapy and open surgery [4].

Radiotherapy alone or in combination with chemotherapy has proven to be an effective treatment modality of laryngeal cancer in early and advanced stages. Early laryngeal cancers are treated with radiotherapy alone [5, 6], while advanced cancers require a combination of both radiotherapy and chemotherapy [7], with an adverse impact on the voice caused by glottic cancers [8].

There is only one study comparing radiotherapy to open surgery. However, interpretation of the results is limited due to concerns about the study methodology and adequacy of treatment regimens.

\section{Case Presentation}

A 59-year-old male patient, a known case of hypertension and type II diabetes mellitus on antihypertensive and oral hypoglycemic agents, with a smoking history of 40 years and a smoking index of 1,600, presented to the emergency department (ED) complaining of inspiratory stridor that started 7 days before seeking medical advice in the ED. The stridor had worsened over time until he sought medical advice. He also had a history of hoarseness of the voice for the past 5 months, weight loss of more than $10 \mathrm{~kg}$ (22 pounds) over the past 6 months, he has no family history of malignancies, and an unremarkable past surgical history. The fiberoptic evaluation in the ED revealed an exophytic ulcerated mass occupying the left vocal cord and partially compromising the airway with fullness of the left pyriform fossa. The right vocal cord could be seen moving. He was admitted to the surgical intensive care unit for airway observation and further evaluation.

Neck and thorax computed tomography (CT) scan with contrast (Fig. 1) showed a heterogeneously enhancing mass lesion in the left side of the larynx predominantly involving the glottic compartment and measuring approximately $1.8 \times 1.5 \times 1.1 \mathrm{~cm}$ in size. The epicenter of this mass lesion appeared to be in the left vocal cord, which extended anteromedially and caused partial compromise of the airway. There was an extension of the lesion into the left aryepiglottic fold. Superiorly, this lesion appeared to extend into the supraglottic region and reaching up to the lower part of the left pyriform sinus. Inferiorly, the mass lesion extended 
Fig. 2. Intraoperative image of the mass as it appears under micro-laryngoscopy.

Fig. 3. Low-power view of the tumor composed of pleomorphic malignant spindle cells. Note the overlying nonatypical squamous mucosa on the top right aspect of the image $(\mathrm{HE}, \times 10)$.
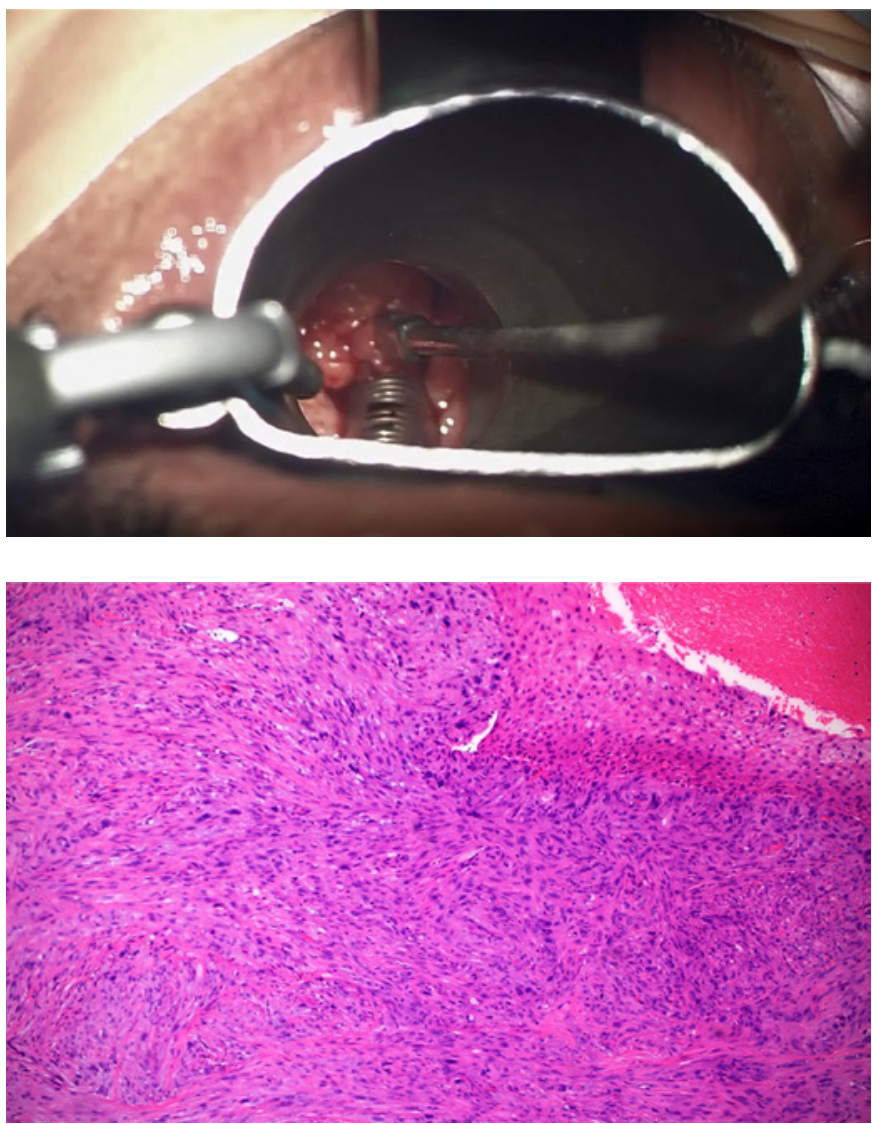

into the upper part of the infraglottic compartment. Laterally, there was extralaryngeal extension into the paralaryngeal space seen abutting the thyrohyoid membrane.

The patient underwent micro-laryngoscopy (Fig. 2) with biopsy and debulking of the mass. Intraoperatively, the tumor was fungating, arising from the supraglottic region; it involved the left vocal cord, sparing the anterior commissure, left arytenoid, left pyriform fossa, and the far posterior aspect of the left vocal fold. Specimen histopathology showed "fragments of partly ulcerated squamous mucosa with extensive underlying infiltration by a malignant neoplasm, composed of pleomorphic spindle cells, interspersed by numerous histiocytes." The malignant spindle cells exhibit frequent mitosis, including atypical mitosis; immunohistochemical stains were positive for smooth muscle actin (SMA), vimentin, and CD68 (in histiocytes), findings consistent with undifferentiated pleomorphic sarcoma (Fig. 3, 4).

Metastases workup was carried out and showed no signs of distant metastasis; the treatment plan was discussed by the multidisciplinary team, and a decision was made to do total laryngectomy with postoperative radiotherapy. The patient underwent total laryngectomy with neck dissection. Frozen sections were done; all margins were negative for malignancy. He received 6 cycles (sessions) of radiotherapy (50-60 Gy) each. On 1-year follow-up, there were no signs of local or distant disease recurrence.

On pathology, the debulked fungating tumor showed partly ulcerated squamous mucosa with an extensive underlying infiltration by a pleomorphic malignant spindle cell neoplasm. It exhibited frequent mitoses; there was no surface squamous dysplasia and no histologic evidence of the tumor originating from the surface. The tumor was subjected to a wide panel 
Fig. 4. High-power view of the tumor showing marked nuclear pleomorphism with frequent mitoses (black arrow) (HE, ×40).

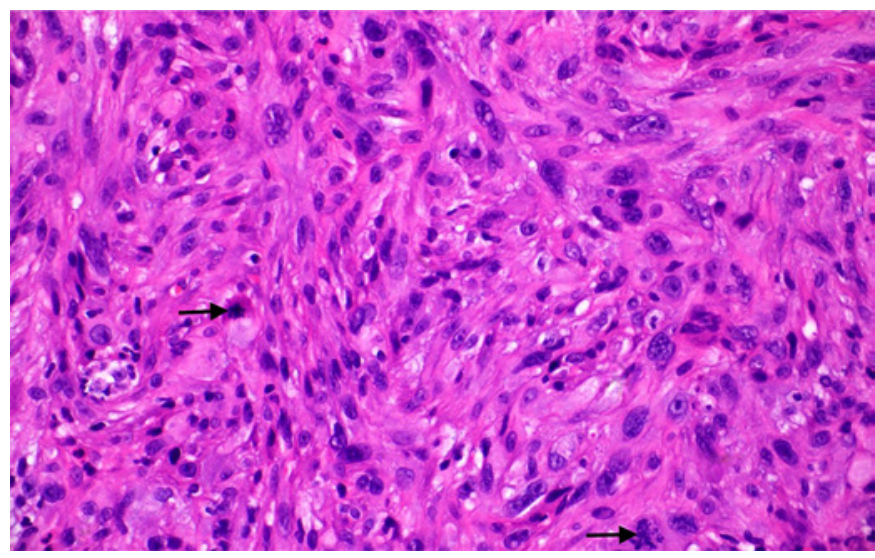

of immune stains, but no definite cell lineage was identified. Areas of SMA and vimentin positivity were present, but various other markers, including cytokeratins, P40, P63, desmin, CD34, CD31, MyoD1, WT1, calretinin, Sox10, S100, MDM2, and CDK4, were all negative. Scattered CD68 positivity highlighted histiocytes interspersed among the tumor cells; the morphological features, coupled with the lack of specific immunohistochemical markers, were consistent with a diagnosis of undifferentiated pleomorphic sarcoma.

Examination of the subsequent laryngectomy specimen revealed residual Grade 3 (following the French Federation of Cancer Centers Sarcoma Group [FNLCC] grading system) tumor in the left vocal cord, measuring $1.5 \mathrm{~cm}$ in maximum dimension, with negative margins.

\section{Discussion}

Sarcomas are rare and complex soft tissue tumors of mesenchymal origin; undifferentiated pleomorphic sarcoma, known also as malignant fibrous histiocytoma (MFH), is the most prevalent type in adults; the incidence is high in the trunk and upper and lower limbs in patients between 50 and 70 years of age [9-11]. The median age of head and neck sarcoma incidence is 55-59 years [12]. Head and neck sarcomas are uncommon, with laryngeal sarcoma considered as a rare tumor, and not many cases are reported in the literature [9]. Sarcomas have different arrays of clinical presentation which varies from small slow-growing lesions to invasive ulceration and wide histologic subtypes [13].

Usually, clinical presentation depends on the site of the primary lesion and growth extent, hoarseness of the voice being the first presenting symptom in tumors originating in the larynx. Dyspnea and stridor are late symptoms, while dysphagia is unlikely unless the tumor extends into the hypopharynx [13].

TNM staging of head and neck sarcomas differs from sarcomas that arise in other parts of the body [14]. Histopathologic grade plays a significant prognostic role in soft tissue sarcomas [15]. Head and neck sarcomas carry a worse prognosis and survival rates lower than 5 years compared to extremities and superficial trunk sarcomas as shown in one singleinstitution series [13]. Laryngeal sarcomas carry a better prognosis than other head and neck sarcomas in general [16], but multiple factors were identified to play a role in overall survival and prognosis.

Head and neck sarcoma treatment varies; surgical excision with or without postsurgical chemoradiotherapy or chemoradiotherapy alone is chosen depending on multiple tumors and patients' related factors. Achieving a complete resection with a negative margin in head 
and neck sarcomas might be difficult due to narrow anatomic spaces and proximity to vital neurovascular structures [17]. A single study in 10 patients with laryngeal sarcomas, where 9 of them were treated with surgery and 1 patient received the full course of radiotherapy due to early diagnosis and old age at presentation, patients treated with surgery showed no evidence of disease recurrence and a 5-year disease-specific survival of 90\% [17].

Management of laryngeal sarcomas will depend mainly on biologic behavior and histologic subtype of the primary tumor, while sarcomas that tend to present as a slowly growing pedunculated mass are less likely to infiltrate into deep tissue, which makes them amenable to surgery within a longer period after diagnosis, unlike squamous cell carcinoma [17]. MFH is a high-histologic-grade tumor and usually carries a poor prognosis. Moreover, high-grade tumors tend to have disseminated disease even without evidence of local recurrence, hence adjuvant chemoradiotherapy is warranted in the treatment plan [18].

Surgical intervention remains superior to any other interventional modality used in laryngeal sarcoma management as shown in a systemic review of laryngeal sarcoma [16]. In our patient, adjuvant chemoradiotherapy was given due to the aggressive nature of the histologic subtype and to minimize the chances of local or distant metastasis; surgery combined with adjuvant chemoradiotherapy will improve the survival rate in patients with laryngeal MFH [19].

\section{Conclusion}

Rare tumors represent a challenge in management due to lack of data in the literature. Unpredicted behavior of these tumors warrants a careful treatment plan considering their indolent nature and possible recurrence and metastasis. With the absence of local recurrence, the management plan should be done through retrospective analysis of outcomes in similar circumstances when available, and the plan of care must consider the best interest of the patient and should include both surgeons and oncologists; in such cases, more is the best.

\section{Acknowledgement}

We thank the patient for letting us share his case. Open Access funding was provided by the Qatar National Library.

\section{Statement of Ethics}

The article describes a case report. Therefore, no additional permission from our Ethics Committee was required. Written informed consent was obtained from the patient's legal guardian for publication of this case report and any accompanying images.

\section{Conflict of Interest Statement}

The authors have no conflicts of interest to declare.

\section{Funding Sources}

This study was not funded.

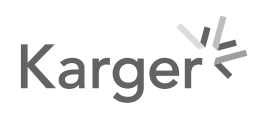




\section{Case Reports in Oncology}

Case Rep Oncol 2021;14:641-646

\section{Author Contributions}

A.A.A.: data collection, literature search, manuscript preparation; A.R.A., H.A.H., W.R., H.A.A.: manuscript preparation; M.P.: pathology slides preparation; A.J.N.: manuscript revision and submission. All authors read and approved the final manuscript.

\section{References}

1 Armstrong WB, Vokes DE, Maisel RH. Malignant tumors of the larynx. In: Flint P, Haughey B, Lund V, Robbins $\mathrm{K}$, Thomas JR, Lesperance M, et al. Cummings otolaryngology, head and neck surgery. Philadelphia: Mosby Elsevier; 2010. p. 1482-511.

2 Bray F, Ferlay J, Soerjomataram I, Siegel RL, Torre LA, Jemal A. Global cancer statistics 2018: GLOBOCAN estimates of incidence and mortality worldwide for 36 cancers in 185 countries. CA Cancer J Clin. 2018;68(6): 394-424.

3 Coleman MP, Estève J, Damiecki P, Arslan A, Renard H. Trends in cancer incidence and mortality. IARC Sci Publ. 1993(121):1-806.

4 Warner L, Chudasama J, Kelly CG, Loughran S, McKenzie K, Wight R, et al. Radiotherapy versus open surgery versus endolaryngeal surgery (with or without laser) for early laryngeal squamous cell cancer. Cochrane Database Syst Rev. 2014(12):CD002027.

5 Agarwal JP, Baccher GK, Waghmare CM, Mallick I, Ghosh-Laskar S, Budrukkar A, et al. Factors affecting the quality of voice in the early glottic cancer treated with radiotherapy. Radiother Oncol. 2009;90(2):177-82.

6 Bibby JR, Cotton SM, Perry A, Corry JF. Voice outcomes after radiotherapy treatment for early glottic cancer: assessment using multidimensional tools. Head Neck. 2008;30(5):600-10.

7 Fung K, Lyden TH, Lee J, Urba SG, Worden F, Eisbruch A, et al. Voice and swallowing outcomes of an organpreservation trial for advanced laryngeal cancer. Int J Radiat Oncol Biol Phys. 2005;63(5):1395-9.

8 Hecker MH, Kreul EJ. Descriptions of the speech of patients with cancer of the vocal folds. I. Measures of fundamental frequency. J Acoust Soc Am. 1971;49(4B):1275-82.

9 Sarcoma Meta-analysis Collaboration (SMAC). Adjuvant chemotherapy for localised resectable soft tissue sarcoma in adults. Cochrane Database Syst Rev. 2000;(2):CD001419.

10 Kim JP, Kim JY, Ko GH, Woo SH. A rare case of malignant fibrous histiocytoma (pleomorphic undifferentiated sarcoma NOS) of the vocal fold. Ear Nose Throat J. 2015;94(7):270-2.

11 Pathrose G, John NT, Manojkumar R. A rare case of malignant fibrous histiocytoma/ pleomorphic undifferentiated sarcoma of the kidney. J Clin Diagn Res. 2015;9(1):PD27.

12 Peng KA, Grogan T, Wang MB. Head and neck sarcomas: analysis of the SEER database. Otolaryngol Head Neck Surg. 2014;151(4):627-33.

13 Zagars GK, Ballo MT, Pisters PW, Pollock RE, Patel SR, Benjamin RS, et al. Prognostic factors for patients with localized soft-tissue sarcoma treated with conservation surgery and radiation therapy: an analysis of 1225 patients. Cancer. 2003;97(10):2530-43.

14 Ługowska I, Raciborska A, Kiprian D, Rysz M, Krajewski R, Świtaj T, et al. Recommendations in management of head and neck sarcomas. Oncol Clin Pract. 2018;14(6):295-301.

15 Cates JM. Performance analysis of the American Joint Committee on Cancer 8th edition staging system for retroperitoneal sarcoma and development of a new staging algorithm for sarcoma-specific survival. Ann Surg Oncol. 2017;24(13):3880-7.

16 Forastiere AA, Ismaila N, Lewin JS, Nathan CA, Adelstein DJ, Eisbruch A, et al. Use of larynx-preservation strategies in the treatment of laryngeal cancer: American Society of Clinical Oncology clinical practice guideline update. J Clin Oncol. 2018;36(11):1143-69.

17 Liu CY, Wang MC, Li WY, Chang SY, Chu PY. Sarcoma of the larynx: treatment results and literature review. J Chin Med Assoc. 2006;69(3):120-4.

18 Pellitteri PK, Ferlito A, Bradley PJ, Shaha AR, Rinaldo A. Management of sarcomas of the head and neck in adults. Oral Oncol. 2003;39(1):2-12.

19 Ferlito A, Nicolai P, Recher G, Narne S. Primary laryngeal malignant fibrous histiocytoma: review of the literature and report of seven cases. Laryngoscope. 1983;93(10):1351-8. 